\title{
Study adherence in a tDCS longitudinal clinical trial with people with spinal cord injury
}

\author{
Sandra Carvalho ${ }^{1,2} \cdot$ Jorge Leite $\mathbb{1}^{1,2,3} \cdot$ Felipe Jones $^{1} \cdot$ Leslie R. Morse $^{4,5,6} \cdot$ Ross Zafonte $^{4,7} \cdot$ Felipe Fregni ${ }^{1,7}$
}

Received: 12 May 2017 / Revised: 29 August 2017 / Accepted: 18 September 2017 / Published online: 13 December 2017

(c) International Spinal Cord Society 2018

\begin{abstract}
Study design Secondary analysis of a clinical trial.

Objectives To analyze adherence to 1-year transcranial Direct Current Stimulation (tDCS) clinical trial in people with chronic pain due to spinal cord injury (SCI). We also explore the association between dropout and several baseline variables such as age, depression levels, pain severity, number of days with pain in the last 7 days, walking ability, sleep, work, relationship with others, and enjoyment with life.

Setting Boston, USA.

Methods Forty-six participants were enrolled in this trial, and 33 participants were randomized to receive either active or sham tDCS.

Results Using the full intention-to-treat (ITT) criteria, only 8 participants (24\%) finished the study. The median time to dropout was seven (IQR:6,19) sessions (i.e., immediately after the first follow-up), regardless of the type of stimulation that participants received (active vs. sham tDCS) $\left(\chi_{2}=0.025, p=0.875\right)$. An exploratory analysis suggested that only the number of days with pain in the last 7 days was moderately associated with dropout, with people experiencing less pain being more prone to dropout from the study.

Conclusions Despite all the measures to improve study adherence (such as providing parking, flexibility to schedule sessions, follow-up with participants by phone), it seems that long follow-up periods may increase the likelihood of dropout. Given the need to understand long-term effects of interventions, longitudinal trials need to consider alternative designs or methods of treatment (for instance home treatment or home assessment) to decrease attrition rate.
\end{abstract}

\section{Introduction}

People with spinal cord injury (SCI) frequently report chronic neuropathic pain that is often refractory to medications $[1,2]$. The development of novel pain therapeutic approaches in the field of SCI research is a priority as laid

Felipe Fregni

fregni.felipe@mgh.harvard.edu

1 Spaulding Neuromodulation Center, Spaulding Rehabilitation Hospital, Department of Physical Medicine and Rehabilitation, Harvard Medical School, Boston, MA, USA

2 Neuropsychophysiology Laboratory, CiPsi, School of Psychology, University of Minho, Campus de Gualtar, Braga, Portugal

3 Univ Portucalense, Portucalense Institute for Human Development -INPP, Oporto, Portugal down by a joint position statement of the US Institutes of Medicine and the National Academy of Sciences. Thus, non-pharmacological interventions such as transcranial Direct Current Stimulation (tDCS) can be helpful for reducing neuropathic pain due to SCI.

Spaulding-Harvard Spinal Cord Injury System, Spaulding Rehabilitation Hospital, Harvard Medical School, Boston, MA, USA

5 Rocky Mountain Regional Spinal Injury System, Craig Rehabilitation Hospital, Englewood, CO, USA

6 Department of PMR, University of Colorado School of Medicine, Aurora, CO, USA

7 Department of Physical Medicine and Rehabilitation, Spaulding Rehabilitation Hospital, Massachusetts General Hospital, Harvard Medical School, Boston, MA, USA 
The number of clinical studies using tDCS has steadily increased in the last years due to its promising clinical results, safety profile, low cost, and relatively easy to use. When applied for sufficient duration, tDCS alters the level of cortical excitability and modulates the firing rate of individual neurons, during and beyond the period of stimulation [3].

Substantial previous research has shown that anodal tDCS over the primary motor cortex (M1) can induce clinically significant pain relief in chronic pain syndromes [4-6] that outlast the stimulation period for several weeks [7-9], with mild or absent side effects [10]. However, these clinical trials often require large sample sizes, strict inclusion, and exclusion criteria, and require multiple study visits per trial. In fact, it has been shown recently that the response to tDCS in fibromyalgia was achieved in $50 \%$ of participants only after 15 sessions [11]. In fact, in our study, in which we tested the effects of M1 tDCS in participants with neuropathic pain following SCI, we showed that a delayed treatment response at 1-week follow-up (after 5 consecutive days of stimulationphase 1) and 4-weeks delayed response after additional 15 days of stimulation (phase 2) [12]. Therefore, the need to have longer periods of treatment together with limitation of transportation of people with SCI pose several potential problems to subject retention, a challenge reflected in trial dropout rates.

Indeed, reasons for dropouts are treatment related; either because the participants were randomized to the active treatment group and experienced adverse side effects, or to the control group and experienced lack of efficacy [13]. Since two meta-analysis have shown significantly higher dropout rates in the placebo arm as compared to the active arm [14], and adverse effects are associated also with increased dropout from a study, here we are interested in analyzing the data comparing active vs. sham groups.

Therefore, in this article, we present adherence data, such as time for dropout, from one longitudinal clinical trial that was designed to assess the efficacy of repetitive sessions of active vs. sham tDCS on pain relief in participant with chronic neuropathic pain secondary to SCI [12]. Since meta-analysis have shown significantly higher dropout rates in the placebo arm as compared to the active arm [14], here we are interested in analysing the data in an exploratory manner comparing active vs. sham group.

Moreover, this specific population may have some specific challenges for adherence; for instance, some characteristics such as severity of lesion (that would result in additional transportation difficulties) may be associated with dropout rates. Thus, in this trial, we further explore this issue, by testing the association between several baseline characteristics such as age, depression levels, pain severity, number of days with pain in the last 7 days, walking ability, sleep, work, relationship with others and enjoyment with life with participant's propensity to dropout from the trial.

\section{Methods}

The data presented in this study is the adherence of people with SCI to a long-term clinical trial (SCIMS trial; clinicalTrials.gov Identifier: NCT01599767), which primary objective was to assess the efficacy of tDCS on pain relief. For more details about the effectiveness of tDCS on pain relief please see Thibaut et al. [12].

\section{Participants}

Forty-six participants (10 females) with SCI (based on the American Spinal Injury Association-ASIA-Impairment Scale performed at time of enrollment) with a mean age of 48 (12.88) with moderate to severe sublesional chronic pain (average Visual Analog Scale (VAS) score of 4 or greater) and time (in years) since injury $6( \pm 6.27)$ ), with self-reported pain since injury, were enrolled for the SCIMS trial (inclusion criteria). From those, 33 participants were randomized to receive either active $(n=16)$ or sham tDCS $(n=17)$.

The exclusion criteria in the study were: active alcohol or drug dependence; history of bipolar disorder or psychosis; inability to travel to the study site; current use of any of the following anti-epileptic medications or dopaminergic medications known to reduce or inhibit the benefits of tDCS treatment: carbamazepine, oxcarbazepine, phenytoin, ropinirole (Requip), pramipexole (Mirapex), and cabergoline (Dostinex); the following contradictions to tDCS: implanted metal plates in the head, or deep brain stimulator (spinal cord implants, including baclofen pumps, are not a contraindication as cranial currents do not reach the spinal cord). Pregnancy at time of enrollment; and current use of ventilator.

All participants provided written informed consent at enrollment and the study was conducted according to the declaration of Helsinki.

\section{Study design}

The complete SCIMS trial involves a total of 21 visits. These visits include, one baseline assessment, 15 daily sessions of tDCS and five follow-up assessments. Of these 21 visits, 4 of the follow-up assessments can be done over the phone, whereas the other 17 visits require the subject to physically travel to the research facility. However, as the duration of this study is quite long (approx. 6 months), the study is divided into two phases, in which the subject is required to consent twice. The first consent is for phase I 


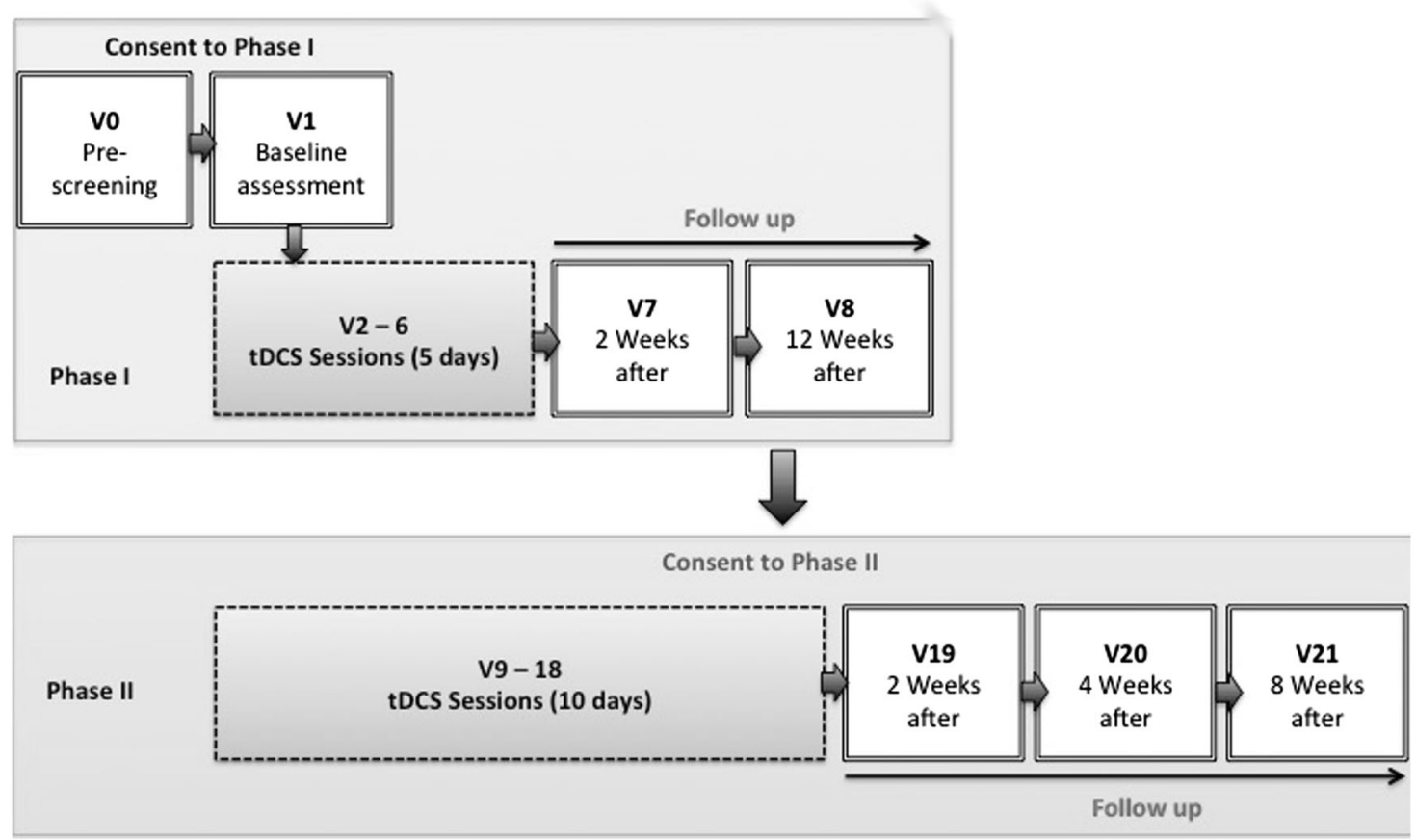

Fig. 1 Study design. Phase 1, from Pre-screening (Visit 0) to follow-up (visit 8); and Phase II, from 6th tDCS session (Visit 9) to follow-up (Visit 21)

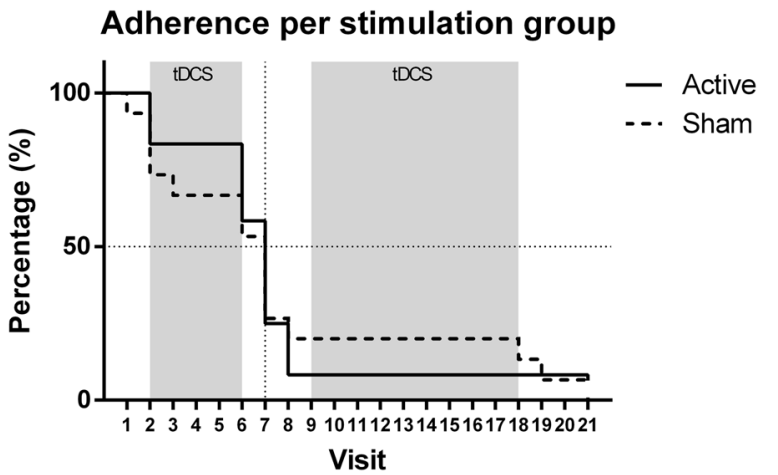

Fig. 2 Participant adherence rate of the SCIMS trial-cumulative retention distributions. tDCS visits are highlighted in gray

(visits 1-8) and if participants wish to continue (and continue to meet eligibility), they may consent for phase II (visits 9-21) (Fig. 1). After a screening assessment to evaluate eligibility for participation, baseline assessments are performed, and participants then undergo five consecutive days of tDCS, with a follow-up at least 1-week post-stimulation. After completion of this first phase, participants have another follow-up visit at least 3 months after the 1-week follow-up. This follow-up visit assesses pain as well as other study measures (such as quality of life and mood). If the subject wishes to continue further into the trial and remains eligible, he/she then consents to the second part of the trial (phase II).
During phase II of the trial, participants received 2-weeks of the tDCS, and are asked to complete three additional follow-up assessment visits ( 2 weeks, 4 weeks, and 8 weeks after their final tDCS session). These three assessments can be done in person, by mail or by phone. All tDCS sessions were performed using the same parameters as in previous studies e.g., ref. [15]. Each stimulation visit lasts about 30 min (20 min of tDCS) and assessment visits may vary from approximately $1-1.5 \mathrm{~h}$.

All enrolled participants were compensated for their time and transportation costs when necessary.

\section{Statistical analysis}

The main objective of the present work was to test if there were differences in term of dropout rate between active and sham tDCS. For that purpose, Kaplan-Meier survival estimates were generated to show the study adherence rates of the two treatment groups (active vs. sham tDCS) throughout the study visits for each respective study. Moreover, the log-rank test was used to evaluate the equality of the survival distributions when comparing the two groups (active and sham tDCS).

The secondary objective was to assess if there were some participant's characteristics that may be associated with their propensity for dropping out from the study. Thus, point biserial correlations were performed in order to assess the potential relationship between dropout and several 
Table 1 Side effects for each group in the SCIMS trial

\begin{tabular}{|c|c|c|c|c|c|c|c|c|c|}
\hline \multirow[b]{2}{*}{ Side effect } & \multicolumn{4}{|c|}{ Sham $(n=17)$} & \multicolumn{5}{|c|}{ Active $(n=16)$} \\
\hline & Absent & Mild & Moderate & Severe & Absent & Mild & Moderate & Severe & Significance \\
\hline Headache & 88.7 & 10.4 & 0.9 & - & 91.9 & 8.1 & 0 & - & $>0.05$ \\
\hline Neck pain & 98.1 & 0.9 & 0.9 & - & 92.6 & 4.4 & 2.2 & - & $>0.05$ \\
\hline Scalp pain & 99.1 & 0.9 & - & - & 92.6 & 5.9 & 1.5 & - & $0.036^{*}$ \\
\hline Scalp burn sensation & 100 & - & - & - & 91.9 & 5.1 & 2.9 & - & $0.006^{*}$ \\
\hline Tingling & 56.1 & 41.1 & 1.9 & 0.9 & 34.6 & 50.7 & 14 & 0.7 & $<0.001 *$ \\
\hline Redness & 83.2 & 15.9 & 0.9 & - & 56.7 & 41.1 & 2.2 & - & $<0.001 *$ \\
\hline Sleepiness & 83.2 & 15.9 & 0.9 & - & 56.7 & 41.1 & 2.2 & - & $<0.001 *$ \\
\hline Concentration & 99.1 & 0.9 & - & - & 100 & - & - & - & $>0.05$ \\
\hline Mood & 100 & - & - & - & 100 & - & - & - & $>0.05$ \\
\hline
\end{tabular}

Values are expressed as a percentage. ${ }^{*}$ Significant at $\mathrm{p}<0.05$ baseline variables such as age, depression levels, pain severity, number of days with pain in the last 7 days, walking ability, sleep, work, relationship with others and enjoyment with life.

In order to assess if there were side effect differences that could explain the dropout rates, Chi-squares tests were performed for each side effect variable in order to assess differences across groups. Fischer's exact tests were performed for groups with less than five observations.

\section{Results}

The median survival until dropout for both groups was 7 (IQR: 6-19) sessions (that indicated the first follow-up). There were no significant differences between the groups (active vs. sham tDCS) in terms of time to dropout $\left(\chi^{2}(1)\right.$ $=0.025, p=0.88$ ) (Fig. 2).

We performed an additional analysis to assess if certain baseline variables were associated with dropout. Therefore, we assessed if age, depression levels, pain severity, number of days with pain in the last 7 days, walking ability, sleep, work, relationship with others, and enjoyment with life were associated with dropout. Only the number of days with pain in the last 7 days was moderately associated with dropout, with people experiencing less pain more prone to dropout from the study $(\mathrm{rpb}=-0.46)$.

\section{tDCS side effects}

Additional analyses were performed to test possible side effects of tDCS on both groups, active vs. sham group. Significant differences were found across groups for side effects of scalp pain $(p=0.036)$, scalp burning sensation $(p=0.006)$, tingling $(p<0.001)$, skin redness $(p<0.001)$, and sleepiness $(p<0.001)$ (Table 1$)$.

\section{Discussion}

This study assessed participants' retention over a longitudinal tDCS clinical trial for pain relief in a cohort of 46 people with SCI.

Overall, the results show that there are no significant differences between active and sham tDCS groups in terms of time to dropout. These results differ from what was previously reported in past trials, namely in meta-analysis that showed that the dropout rates in the placebo arm were significantly higher than the active arm [14]. Moreover, a recent meta-analysis assessing the dropout rate in antidepressant studies using placebo or active comparator, suggested that the dropout rate in the placebo arm was strongly associated with the lack of efficacy [16], despite no significant difference dropout rate between active $(24.3 \pm$ $8.27)$ vs. placebo $(24.0 \pm 9.04 \%)$ for the trials conducted in the last decade. The reason for the differences here may also be because of better placebo methods for tDCS, which seems to be less guessed as active when compared with drugs [17]. Nonetheless, it is important to highlight that the sample size of the present study was rather small, and as such the lack of a significant difference in terms of adherence to active or sham tDCS needs to be confirmed by larger trials as this may be a type II error.

In the present trial, only 8 (out of 33) participants completed the trial, which translates to a dropout rate of $76 \%$. A recent 16-week long duration trial assessing the effectiveness of physical activity guidelines in patients with SCI, showed a $18 \%$ dropout rate [18]. This rate was slightly below the one found in other chronic pain trials, in which dropout rates range from 20 to $50 \%$ [13]. Moreover, usually the reasons for dropouts are treatment related; either because the participants were randomized to the active treatment group and experienced adverse side effects, or to the control group and experienced lack of efficacy [13]. 
The results from this exploratory study show that tDCS had a delayed pain decrease at 1-week follow-up for phase 1 and only at 4-week follow-up for phase II [12]. This delayed response to intervention may potentially explain the loss to follow-up in this trial, since participants may perceived this intervention as not working as expected or they believed they were receiving sham tDCS.

Another important aspect to consider in this perceived lack of efficacy is the definition of the maximum pain relief that the intervention is able to induce, or simply what do participants perceive as pain relief. A recent study with chronic neuropathic pain following fibromyalgia suggested that 15 sessions may be the median number of sessions required in order to induce a significant clinical effect (i.e., $50 \%$ pain reduction) [11]. Thus, it may be possible that five consecutive sessions were not able to induce sufficient pain relief in this population to be perceived as such up to that point. This perceived lack of efficacy could potentially explain the dropout of participants following the first round of $\mathrm{tDCS}$.

Another possibility for this high dropout rate could be the potential side effects that arose from the intervention. This has been already shown extensively in the literature, in which increased dropout rate in drug trials is significantly associated with adverse effect severity [19, 20]. This could certainly be the case in this study, as participants with SCI submitted to verum stimulation reported significant more skin redness, sleepiness, scalp pain, scalp burning sensation, and tingling as compared to those receiving the placebo. However, when these side effects were reported, most of them were of mild severity-which is consistent with previous reports in the literature [21]. Thus, side effects do not seem to have influenced adherence in the present trial. Moreover, the difference in terms of absolute dropout rate between groups is inferior to $15 \%$, which suggests a low risk of intervention induced bias. It has also been suggested that trial design, type of intervention, geographic area, or culture can contribute for participants' dropout rate [14]. Mobility difficulties may also have impacted the adherence of these participants to the clinical trial. In fact, a multicentre trial with people with SCI demonstrated an inverse relationship between mobility and pain scores, i.e., people with moderate to high levels of mobility reported less pain [22]. Moreover, people with chronic pain and SCI have high susceptibility to stress and depression [23, 24], which can seriously affect their adherence to time demanding clinical trials. All of these factors may increase burden to participants, and thus contribute to study dropout. Indeed, adherence in this specific population may be predicted more by burden related to research procedures (i.e., visits and/or travel) than by efficacy of treatment. For instance, a previous trial showed that repeated administration of
onabotulinumtoxinA detrusor injections every 6 months improved their incontinence problems, but despite that, only $20 \%$ of patients continued the repeated treatment [25].

In the present study, there were no differences between active and sham tDCS in terms of participants' propensity to dropout, but there was an overall high dropout rate. Therefore, we performed additional analysis to assess if certain baseline variables, such as age, depression levels, pain severity, number of days with pain in the last 7 days, walking ability, sleep, work, relationship with others and enjoyment with life, were associated with dropout. Results show that only the number of days with pain in the last 7 days was moderately associated with dropout, with people experiencing less pain being more prone to dropout from the study. This is an interesting result, as it seems that pain severity or level of pain seem to affect study adherence. On the other hand, as shown before higher pain at baseline may indicate more refractoriness to tDCS [15]. Future studies should take this in consideration when determining study population, as it seems that people with higher levels of pain are more motivated to travel to the research facility for multiple tDCS sessions.

For those reasons, it is important to offer to these participants, new options such as the possibility of participating in the stimulation and assessment sessions at home with remote assistance from the clinical and research setting. Therefore, the development of home-based protocols can be very useful in order to overcome this difficulty trial compliance [26].

Another interesting result in this trial is that, participants tend to dropout from the study after the screening assessment, prior to actual study commencement, as loss to follow-up slope is greater at that point (see Fig. 1). So, in the absence of perceived early benefits from the intervention, adherence may be suboptimal. Once participants actually start the stimulation, most participants do not dropout until completion of the stimulation sessions. The dropout rates increase again during the follow-up assessment visits. One possibility is that the one that feel greater pain, are also the ones more motivated to be complete the stimulation sessions because of the potential clinical benefits. As soon as therapy ends, then they are less likely to adhere to the assessments that will not be perceived as having any clinical benefit.

This loss to follow-up has been reported in many other clinical trials [27, 28], and poses serious threats to the validity of the study by potentially introducing bias to the results, as participants that dropout may have distinct characteristics from those that do not dropout [29]. Again, it is not possible to disentangle if this high dropout rate was due to lack of perceived efficacy of the tDCS intervention. If the pain levels were low enough for them to dropout from the study, or even if in low levels of pain there is no actual 
benefit from the tDCS intervention or in the case there is, the effects are not perceived as beneficial.

It is important to highlight that in the SCIMS trial, participants were given the opportunity to perform follow-up assessments over the phone. Despite that, there was a significant loss to follow-up, starting at 1 month after tDCS has ended. It is possible that the elapsed time between the stimulation and the follow-up assessments negatively impacted participants' motivation to continue in the trial. Moreover, it is possible that the intervention's perceived lack of efficacy increased over time, as the perceived potential benefits were becoming smaller. Additionally, participants may have felt more motivated to receive the intervention rather than complete the long follow-up assessments (with a 1-hour burden).

Table 2 Recommendations for future longitudinal clinical trials with tDCS for Chronic Pain with people with spinal cord injury

Recommendations for future longitudinal clinical trials with tDCS for SCI

Participant-centered:

Participants with lower levels of pain may be more vulnerable to dropout in long-term clinical trials:

Participants with resistant pain may be more motivated to complete the trial

Motivation to participate in the trial needs to be closely monitored in the different phases of the trial, especially during assessments

Difficulties with mobility may impact adherence

For short-term trials, improving commuting to the research facility may be helpful

For long-term trials, home-based intervention options may be considered, thus reducing the impact on their lives

Participants-directed strategies to improve compliance may include education about the relevance of the clinical trial and about her/his adherence to all regimens (including assessments).

Research team-centered:

Trials should be designed in order to include specific strategies that aim to increase compliance in different phases of the clinical trial:

Well-prepared research team

Good communication skills

Welcoming behavior with participants

Providing flexible or expanded clinic hours.

Uncertain benefits and potential risks associated with participation can impact compliance. This should be discussed and monitored with participants.

Family and friends centered:

Involving family and friends in the treatment may also have a positive impact on compliance by increasing the social support to the subject. Depending on specific personal characteristics and needs of the participant, family and friends can be involved in order to support the subject during his/her participation.
This study is one of the first studies testing the effectiveness of tDCS for chronic neuropathic pain in people with SCI with long follow-ups (that showed to be important given our efficacy results please see ref. [12]. And as such, a placebo intervention (i.e., sham tDCS) was chosen as comparator in order to increase the validity of the results, especially due to the 15 sessions timeframe that has been shown to induce a clinical meaningful effect in terms of pain relief. Thus, in this sense, it was important to maintain the comparator across the 15 sessions. If there seem to be no differences in terms of dropout rates due to comparator, the available data suggest that, future longitudinal tDCS clinical trials with participants with SCI, may benefit from changes in the study design. First of all, patients with refractory pain may be more prone to adhere to the study. Second, at early stages of the intervention adherence may be improved by facilitating the commute to the research facility. But if these trials require larger time commitments, a stepped home intervention may be more beneficial (see Fig. 1). Additionally, one limitation of this study was that subjects were randomized upon consenting, and that may have produced a minor imbalance in groups prior to the start of stimulation. Therefore, future studies should only perform subject's randomization subjects immediately before the first stimulation session.

\section{Conclusion and future directions}

This work provides insights about the participants' adherence to long-term clinical trials assessing the effect of tDCS for pain relief. Despite the safety profile of tDCS and the absent difference in terms of dropout rate between active and sham tDCS groups, long-term clinical trials remain a challenge for research participants. Specific populations may be more vulnerable for this long-term adherence commitment than others, especially those with lower levels of pain. Special attention must be given to long-term follow-ups, especially those that involve a significant time burden for participants. Trials should be designed in order to include specific strategies that aim to increase compliance in different phases of the clinical trial. These patientdirected strategies to improve compliance may include education about the relevance of the clinical trial and about her/his adherence to all regimens (including assessments). Some recommendations to improve adherence are summarized in Table 2.

Acknowledgements This project was supported by the National Institute on Disability, Independent Living, and Rehabilitation Research (NIDILRR grant numbers H133N110010 and 90SI5021-0100). SC and JL are supported by the Portuguese Foundation for Science and Technology PTDC/MHC-PCN/3950/2014; SC is also funded by the following FCT grant IF/00091/2015. 


\section{Compliance with ethical standards}

Conflict of interest The authors declare that they have no conflict of interest.

Disclaimer The authors are responsible for the content and writing of this article.

\section{References}

1. Siddall PJ, McClelland JM, Rutkowski SB, Cousins MJ. A longitudinal study of the prevalence and characteristics of pain in the first 5 years following spinal cord injury. Pain. 2003;103:249-57.

2. Widerstrom-Noga EG, Turk DC. Types and effectiveness of treatments used by people with chronic pain associated with spinal cord injuries: influence of pain and psychosocial characteristics. Spinal Cord. 2003;41:600-9.

3. Nitsche MA, Paulus W. Sustained excitability elevations induced by transcranial DC motor cortex stimulation in humans. Neurology. 2001;57:1899-901.

4. Brietzke AP, Rozisky JR, Dussan-Sarria JA, Deitos A, Laste G, Hoppe PF, et al. Neuroplastic effects of transcranial direct current stimulation on painful symptoms reduction in chronic hepatitis $\mathrm{C}$ : A phase II randomized, double blind, sham controlled trial. Front Neurosci. 2015;9:498.

5. Moreno-Duarte I, Morse LR, Alam M, Bikson M, Zafonte R, Fregni F. Targeted therapies using electrical and magnetic neural stimulation for the treatment of chronic pain in spinal cord injury. Neuroimage. 2014;85:1003-13.

6. Soler MD, Kumru H, Pelayo R, Vidal J, Tormos JM, Fregni F, et al. Effectiveness of transcranial direct current stimulation and visual illusion on neuropathic pain in spinal cord injury. Brain. 2010;133:2565-77.

7. Carvalho S, Goncalves OF, Soares JM, Sampaio A, Macedo F, Fregni F, et al. Sustained effects of a neural-based intervention in a refractory case of Tourette syndrome. Brain Stimul. 2015;8:657-9.

8. Cioato SG, Medeiros LF, Marques Filho PR, Vercelino R, de Souza A, Scarabelot VL, et al. Long-lasting effect of transcranial direct current stimulation in the reversal of hyperalgesia and cytokine alterations induced by the neuropathic pain model. Brain Stimul. 2016;9:209-17.

9. Rostami R, Badran BW, Kazemi R, Habibnezhad M, George MS. Long-lasting analgesic effect of transcranial direct current stimulation in treatment of chronic endometriosis pain. J Obstet Gynaecol Res. 2015;41:1998-2001.

10. Fregni F, Nitsche MA, Loo CK, Brunoni AR, Marangolo P, Leite $\mathrm{J}$, et al. Regulatory Considerations for the clinical and research use of transcranial direct current stimulation (tDCS): review and recommendations from an expert panel. Clin Res Regul Aff. 2015;32:22-35.

11. Castillo-Saavedra L, Gebodh N, Bikson M, Diaz-Cruz C, Brandao $\mathrm{R}$, Coutinho L, et al. Clinically effective treatment of fibromyalgia pain with high-definition transcranial direct current stimulation: Phase II open-label dose optimization. J Pain. 2016;17:14-26.

12. Thibaut A, Carvalho S, Morse LR, Zafonte R, Fregni F. Delayed pain decrease following M1 tDCS in spinal cord injury: A randomized controlled clinical trial. Neuroscience Letters. 2017;658:19-26.
13. Kim Y. Missing data handling in chronic pain trials. J Biopharm Stat. 2011;21:311-25.

14. Kemmler G, Hummer M, Widschwendter C, Fleischhacker WW. Dropout rates in placebo-controlled and active-control clinical trials of antipsychotic drugs: a meta-analysis. Arch Gen Psychiatr. 2005;62:1305-12.

15. Fregni F, Boggio PS, Lima MC, Ferreira MJ, Wagner T, Rigonatti SP, et al. A sham-controlled, phase II trial of transcranial direct current stimulation for the treatment of central pain in traumatic spinal cord injury. Pain. 2006;122:197-209.

16. Schalkwijk S, Undurraga J, Tondo L, Baldessarini RJ. Declining efficacy in controlled trials of antidepressants: effects of placebo dropout. Int J Neuropsychopharmacol. 2014; 17:1343-52.

17. Brunoni AR, Schestatsky P, Lotufo PA, Bensenor IM, Fregni F. Comparison of blinding effectiveness between sham tDCS and placebo sertraline in a 6-week major depression randomized clinical trial. Clin Neurophysiol. 2014;125:298-305.

18. Pelletier CA, Totosy de Zepetnek JO, MacDonald MJ, Hicks AL. A 16-week randomized controlled trial evaluating the physical activity guidelines for adults with spinal cord injury. Spinal Cord. 2015;53:363-7.

19. Kostev K, Rex J, Eith T, Heilmaier C. Which adverse effects influence the dropout rate in selective serotonin reuptake inhibitor (SSRI) treatment? Results for 50,824 patients. Ger Med Sci. 2014;12:1-8

20. Gehling M, Hermann B, Tryba M. Meta-analysis of dropout rates in randomized controlled clinical trials: opioid analgesia for osteoarthritis pain. Schmerz. 2011;25:296-305.

21. Brunoni AR, Nitsche MA, Bolognini N, Bikson M, Wagner T, Merabet L, et al. Clinical research with transcranial direct current stimulation (tDCS): challenges and future directions. Brain Stimul. 2012;5:175-95.

22. Marcondes BF, Sreepathi S, Markowski J, Nguyen D, Stock SR, Carvalho S, et al. Pain severity and mobility one year after spinal cord injury: a multicenter, cross-sectional study. Eur J Phys Rehabil Med. 2016;52:630-6.

23. Brommer B, Engel O, Kopp MA, Watzlawick R, Muller S, Pruss $\mathrm{H}$, et al. Spinal cord injury-induced immune deficiency syndrome enhances infection susceptibility dependent on lesion level. Brain. 2016;139:692-707.

24. Elliott TR. Studying depression following spinal cord injury: Evidence, policy and practice. J Spinal Cord Med. 2015;38: 584-6.

25. Chen SF, Kuo HC. Therapeutic outcome and patient adherence to repeated onabotulinumtoxinA detrusor injections in chronic spinal cord-injured patients and neurogenic detrusor overactivity. J Formos Med Assoc. 2015;114:583-9.

26. Charvet LE, Kasschau M, Datta A, Knotkova H, Stevens MC, Alonzo A, et al. Remotely-supervised transcranial direct current stimulation (tDCS) for clinical trials: guidelines for technology and protocols. Front Syst Neurosci. 2015;9:26.

27. Akl EA, Briel M, You JJ, Sun X, Johnston BC, Busse JW, et al. Potential impact on estimated treatment effects of information lost to follow-up in randomised controlled trials (LOST-IT): systematic review. BMJ. 2012;344:e2809.

28. Vervolgyi E, Kromp M, Skipka G, Bender R, Kaiser T. Reporting of loss to follow-up information in randomised controlled trials with time-to-event outcomes: a literature survey. BMC Med Res Methodol. 2011;11:130.

29. Dettori JR. Loss to follow-up. Evid Based Spine Care J. 2011;2:7-10. 